\title{
r-Process Enhanced Metal-Poor Stars
}

\section{Christopher Sneden*广}

Department of Astronomy, University of Texas at Austin, Austin, TX 78712, USA

E-mail: chris@verdi.as.utexas.edu

\section{Ian U. Roederer}

Carnegie Observatories, 813 Santa Barbara St., Pasadena, CA 91101, USA E-mail:

iroederer@obs.carnegiescience.edu

\section{John J. Cowan}

HLD Dept. of Physics \& Astronomy, University of Oklahoma, Norman, OK 73019, USA

E-mail: cowan@nhn.ou.edu

\section{James E. Lawler}

Department of Physics, University of Wisconsin, Madison, WI 53706, USA

E-mail: jel@wisc.edu

In this review we summarize the current state of some aspects of observational studies of low metallicity stars that have been enriched with products of rapid-neutron-capture ( $r$-process) nucleosynthesis events. Attention will be drawn to the element domains where there is little star-tostar variation in relative abundances (such as the rare earths), and those with substantial variations (the lightest neutron-capture elements and the very heavy radioactive elements $\mathrm{Th}$ and $\mathrm{U}$ ). We highlight some new work bearing on our understanding of the limits of abundance distributions that can be produced in the $r$-process. Finally, comments will be made on the metallicity at which significant contributions from the $s$-process can be seen in Galactic halo stars.

11th Symposium on Nuclei in the Cosmos, NIC XI

July 19-23, 2010

Heidelberg, Germany

\footnotetext{
*Speaker.

${ }^{\dagger}$ Funding for this work has been provided by grants for a number of years to all the authors from the US National Science Foundation.
} 


\section{Introduction}

Elements with atomic numbers $\mathrm{Z}>30$ are synthesized in the late stages of stellar evolution, almost exclusively via neutron-capture (n-capture) reactions with seed nuclei in nuclear fusion interior zones. Two extreme, but apparently realistic, physical situations have been defined for how $n$-capture synthesis works in stars. If successive neutron captures happen slowly compared to $\beta$-decay rates of unstable nuclei, then isotope/element synthesis proceeds along the valley of $\beta$ stability; this is called the s-process. But if the neutron captures flood nuclei much more rapidly than $\beta$-decays can adjust for stability, isotope/element synthesis is skewed to neutron-rich nuclei; this is called the $r$-process.

Fertile astronomical testing grounds for $r$-process and $s$-process physics are the spectra of metal-poor Galactic halo stars that are anomalously enriched in $n$-capture elements. Twenty years ago we knew of only a few such stars, e.g, HD 115444 [8], and thus had no understanding of how widespread the phenomenon might be. Ten years ago some photometric and medium-resolution spectroscopic surveys (e.g., [2]) of Galactic pole regions had provided promising candidates for high-resolution spectroscopic investigation. Today there are hundreds of $r$-rich and $s$-rich stars that have been identified and have enjoyed abundance analyses detailed enough to understand their basic nucleosynthetic mix. A lesser number these stars have been subjected to repeated analyses, so that now we have abundances for nearly every $n$-capture element that can be detected in their spectra.

A general review of $n$-capture-rich very metal-poor stars has been recently published [26], to which the reader is referred for an overview of the field to that time. In this brief paper we propose to highlight a few of the advances in observational studies of $r$-process-rich stars since that review. The subject of $s$-process- and C-rich low metallicity stars deserves its own review; these stars will not be considered further here.

\section{More and Ever-Improved Analyses}

The number of well-studied $r$-rich stars grows steadily. In [26], Figure 11 presented data for six of these stars, comparing their $n$-capture abundances with that of the solar-system $r$-processonly distribution. The excellent matches over the whole $n$-capture atomic number range of stable elements suggests that the same process has created the $r$-process elements over the entire Galactic history.

The ability to produce such detailed matches for large numbers of $n$-capture elements per star (more than 30 in the best case) has been enabled by several factors. Firstly, when $[n$-capture/Fe] $\geq$ $+1, r$-process-rich metal poor stars simply have very large line strength contrasts between the their $n$-capture elements and lighter ones. Secondly, the current generation of $8 \mathrm{~m}$-class telescopes equipped with efficient high-resolution spectrographs has provided high-S/N spectra of these stars over wavelength ranges that stretch down nearly to the ultraviolet atmospheric cutoff, and in a few cases into the vacuum ultraviolet [4]. Thirdly, several atomic physics groups have produced very accurate transition probabilities and hyperfine/isotopic substructure data for large numbers of lines of atomic species that are most useful to spectroscopists of metal-poor stars (e.g, [18, 16, 21]). 


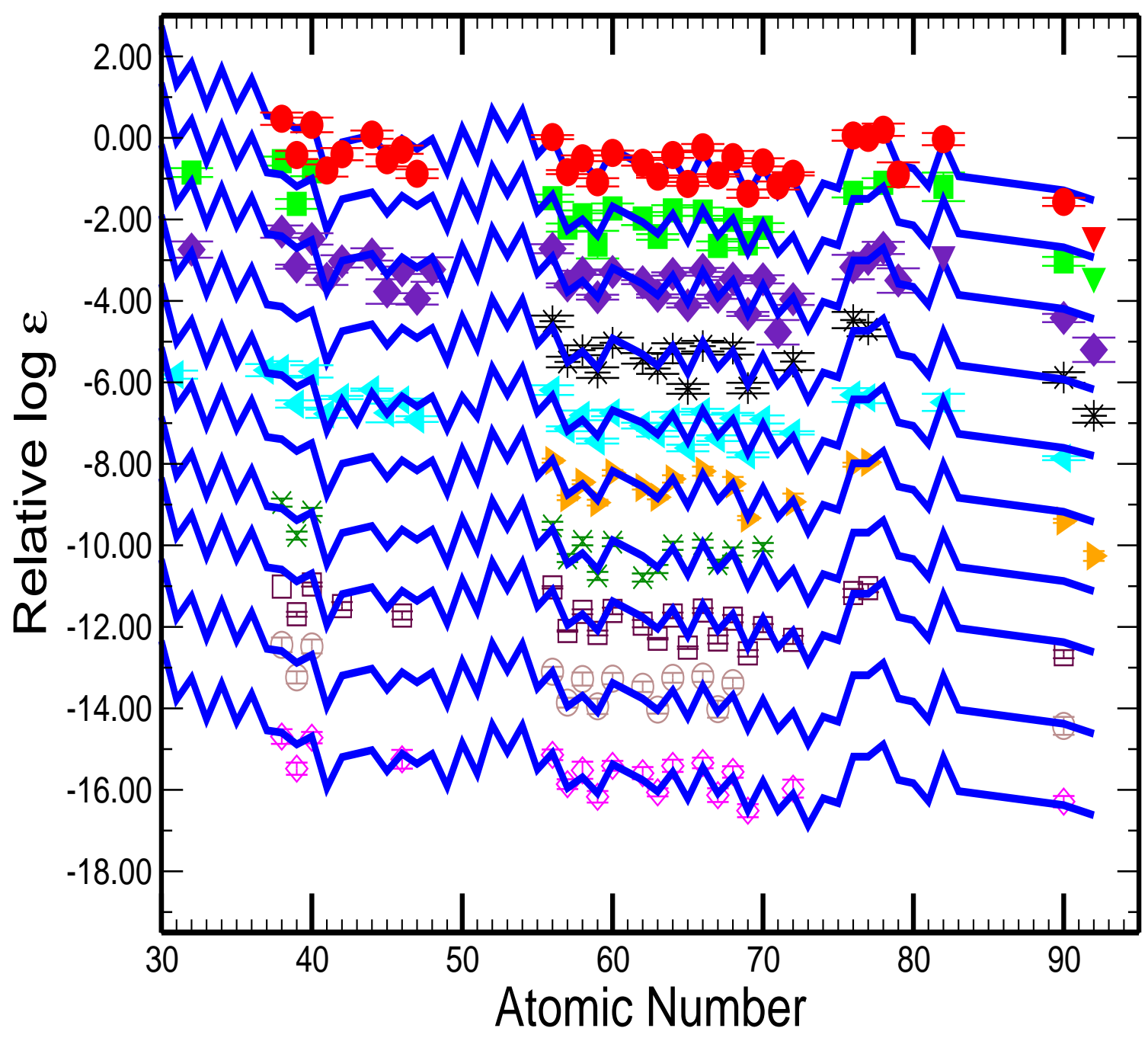

Figure 1: Expansion of the top panel of Figure 11 in [26]. The solid blue lines are the scaled $r$-process-only solar-system elemental abundance curves, normalized to the Eu abundance of each star. Stellar data (from the top) are: filled (red) circles, CS 22892-052 [27, 28], filled (green) squares, HD 115444 [30, 28], filled (purple) diamonds, BD+17 3248 [3, 24], (black) stars, CS 31082-001 [10, 28], solid (turquoise) left-pointing triangles, HD 221170 [13, 28], solid (orange) right-pointing triangles, HE 1523-0901 [6], (green) crosses, CS 22953-003 [5], open (maroon) squares, HE 2327-5642 [20], open (brown) circles, CS 29491-069 [9], open (magenta) triangles, HE 1219-0312 [9], All abundances, except those of CS 22892-052, have been vertically displaced downwards for display purposes. The solid lines are solar system r-process only predictions from [25]. 
The six stars chosen by [26] for relative abundance display hardly exhaust the list of "solar- $r$ " stars among the $n$-capture-enhanced low metallicity Galactic halo population. In Figure 1 we add several more examples, to emphasize that multiple teams of investigators find essentially the same results. Inspection of this figure re-affirms some conclusions that have been reached in many of the cited papers: $(a)$ the match to the solar-system abundances is the best for stable elements in the atomic number domain $56 \leq \mathrm{Z} \leq 78$, i.e, the rare earths and the $3^{r d} r$-process-peak elements; (b) the abundance data of lighter $n$-capture elements are relatively sparse except for the easilyobserved Sr-Y-Zr group, due to the relative weakness of lines of most of these elements in metalpoor stars; (c) among the lighter elements, $Y(Z=39)$ and $\mathrm{Ag}(Z=47)$ persistently show relative underabundances by factors of 2-3 compared to their neighboring elements with respect to the solar $r$-process distribution; and $(d)$ much scatter exists in the abundances of very heavy radioactive elements $\mathrm{Th}(Z=90)$ and $\mathrm{U}(\mathrm{Z}=92)$ with respect to the heavier stable $n$-capture elements, which may compromise their utility as cosmochronometers.

The relative abundance data for many $n$-capture-rich metal-poor stars are now good enough that insights into $r$-process nucleosynthesis may be sought in star-to-star abundance differences at the $<0.1$ dex level. One such exploration has recently been undertaken by [22], a summary of which is among the present conference papers. From that work we have reproduced in Figure 2 a rare-earth abundance comparison between the two best-studied stars with extreme $r$-process enhancements (CS 22892-052 [27, 28] and CS 31082-001 [10, 28]), and three stars with merely very large enhancements (BD+17 3248 [3, 28, 24], HD 115444 [30, 28], and HD 221170 [13, 28]). The differences are subtle: when the curves are normalized at $\mathrm{Eu}$, the three "lightest" elements Ba-La-Ce are $\simeq 0.05-0.10$ more abundant in the less $r$-process-enhanced trio of stars. Since in the solar-system these elements have an overwhelmingly s-process origin, the simplest interpretation is that the difference in the two stellar groups is the addition of a small amount of $s$-process material to an $n$-capture element set that is still has had an overwhelmingly $r$-process origin. However, this idea rests on the assumption that the abundance distribution resulting from an $r$-process event is identical to all others. Increasing evidence supports the notion of star-to-star abundance differences among apparently $r$-rich stars. We explore this idea in the next section.

\section{Evidence for Variety in the $r$-Process}

Careful abundance investigations of the bright very metal-poor, $n$-capture-poor giants HD 122563 and HD 88609 [11, 12] have shown fairly conclusively that not only is the solar-system $r$-process distribution a poor match to the $n$-capture abundances of these stars, but the $s$-process "main" and "weak" fail as well. These authors argue an $r$-process created the $n$-capture elements in these stars, but with different fusion region physical conditions. Some theoretical investigations have now considered varying parameters (such as the electron fraction $\mathrm{Y}_{e}$ [29]). More generally, it is important to remember that theoretical predictions of the solar-system $r$-process distribution (e.g, [14]) fail if they assume a single value of the neutron density in the synthesis regime. Multiple (extremely high) neutron density regimes that range over five orders of magnitude must be invoked in order to match the abundances of light and heavy $n$-capture elements (as demonstrated in Figures 5 and 6 of [14]). 


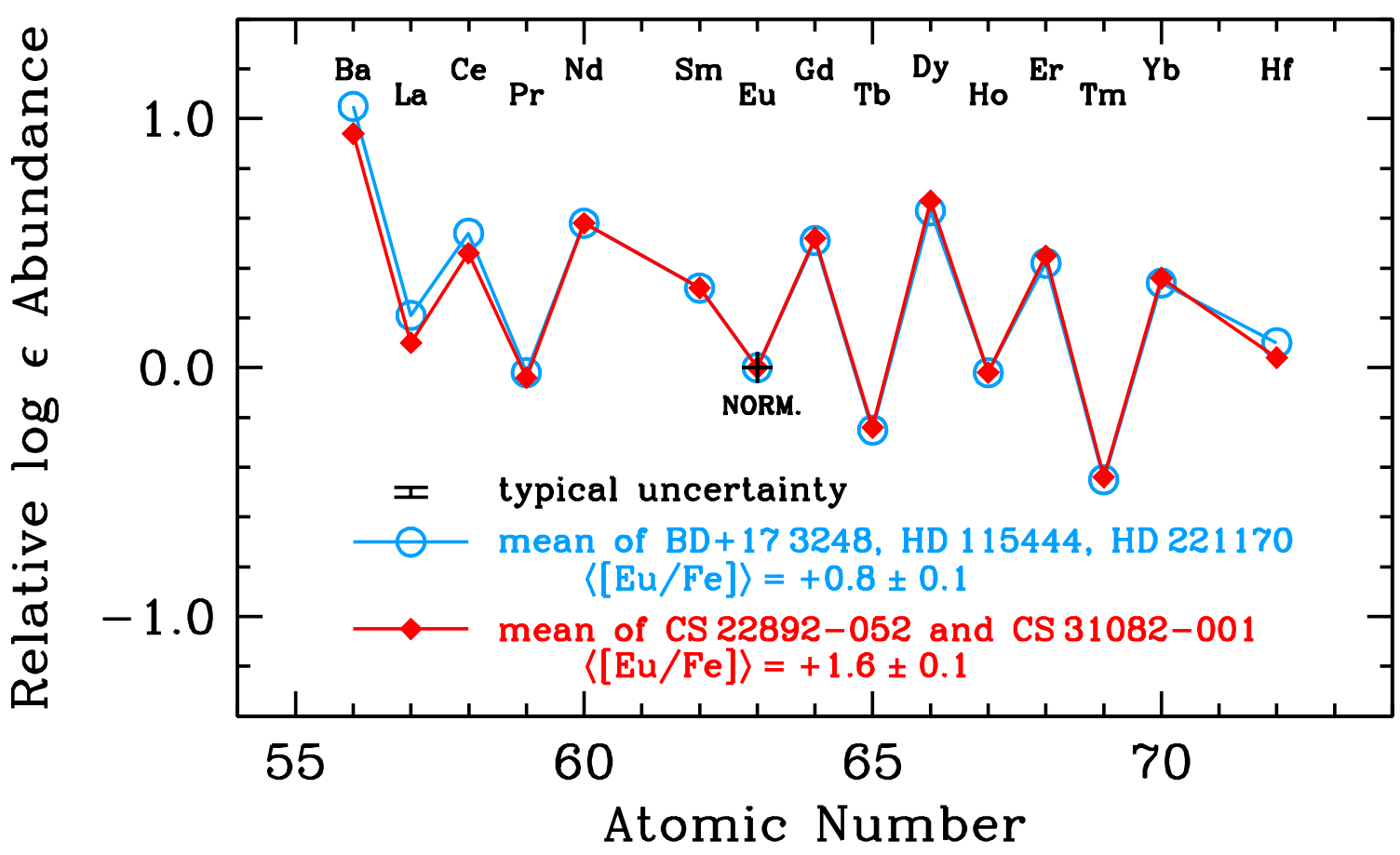

Figure 2: Relative mean abundances for the rare-earth elements in two groups of $r$-process standard stars. The first group (blue circles) has $\langle[\mathrm{Eu} / \mathrm{Fe}]\rangle=+0.8 \pm 0.1$, and the second group (red diamonds) has $\langle[\mathrm{Eu} / \mathrm{Fe}]\rangle=+1.6 \pm 0.1$. The abundances are normalized to $\mathrm{Eu}(Z=63)$. Abundances are taken from [28].

An attempt to define $r$-process synthesis possibilities from observed abundance distributions has recently been completed by [22] (see their contribution to this conference). They argue that the existence of substantial $s$-process nucleosynthesis in metal-poor stellar spectra can be inferred if lead (in the form of the $\mathrm{Pb}$ I $4057 \AA$ line) is detected. This is due to the tendency of the $s$-process to run to the heaviest stable elements at low metallicity (due to the large neutron-seed-nuclei ratios in the He-fusion zones of such stars. With this discriminant, it is possible to identify stars without any evidence of $s$-process enrichment that nevertheless have substantially larger $\mathrm{La} / \mathrm{Eu}$ ratios than those of the the solar-system or such "standard" $r$-rich stars as CS 22892-052. In Figure 3, a reproduction of part of Figure 11 of [22], the variety of $r$-process stars defined in this manner is displayed. This plot includes stars at the extremes of claimed $r$-process distributions that were shown in previous figures, and a variety of intermediate cases. It is difficult to make definitive arguments for $r$-processonly synthesis in cases that depart significantly from the scaled solar-system distribution. Clearly much more work, especially in $r$-process theory, will be needed to understand this situation better.

\section{At What Metallicity Does the $s$-Process Appear?}

A new study of Ba isotopes in the bright metal-poor subgiant HD $140283([\mathrm{Fe} / \mathrm{H}] \simeq-2.6,[7]$, and reported at this conference) argues that the ratio of odd isotopes to even isotopes is consistent with a pure $s$-process origin to the $n$-capture elements in this star. Their analysis is careful and extensive, and appears to be the definitive word on the Ba isotope mix (discussed earlier by $[15,19]$ ) in this star. The other abundances and upper limits of the very few detectable $n$-capture elements 


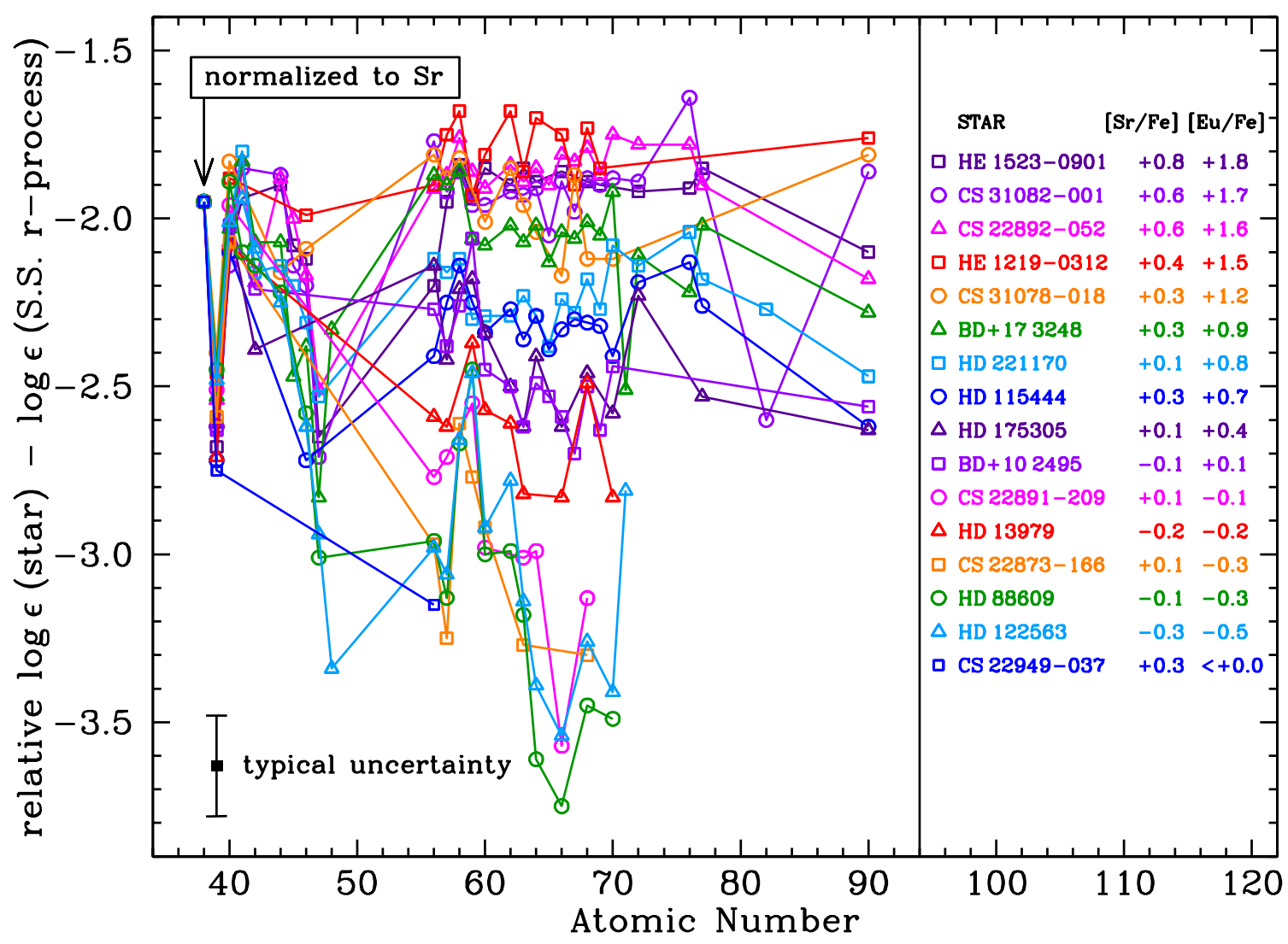

Figure 3: (Part of Figure 11 of [22]) Differences between the solar-system $r$-process abundances and stellar abundances for 16 metal-poor stars, normalized to $\operatorname{Sr}(Z=38)$ to minimize the effect of overall metallicity differences. The stars are listed according to decreasing [Eu/Fe] and are identified, along with their $[\mathrm{Sr} / \mathrm{Fe}]$ and $[\mathrm{Eu} / \mathrm{Fe}]$ ratios, in the box to the right. A typical uncertainty is indicated in the lower left corner. This confirms the conclusion of [12] that the heavy element abundance pattern of CS 22892-052 is distinct from HD 88609 and HD 122563. Furthermore, there is a continuous distribution of abundance patterns in other stars that fall between these two extremes. Please see [22] figure caption for citations to the papers with the original data for each star.

in this very line-weak spectrum are also consistent with an $s$-process interpretation. These data potentially make HD 140283's $n$-capture abundances inconsistent wht prior claims of pure $r$-process nucleosynthesis at the low metallicity of this star, $[\mathrm{Fe} / \mathrm{H}] \simeq-2.6$.

However, work over the last decade has broadened our view of $n$-capture nucleosynthesis in the early Galaxy. In Figure 4 we reproduce Figure 16 of [26], in which La/Eu ratios (in $\log \varepsilon$ units) are correlated with $[\mathrm{Fe} / \mathrm{H}]$. Inspection of the data clearly shows the trend in average $\mathrm{La} / \mathrm{Eu}$ values to decrease with decreasing metallicity, and for $[\mathrm{Fe} / \mathrm{H}]<-2.5$ a number of stars (mostly discussed in detail in earlier sections of this paper) have $\mathrm{La} / \mathrm{Eu}$ ratios consistent with the solar-system $r$-process ratio. But many stars have substantially higher values even at these low metallicities. As we discussed in the previous section, some of these higher La/Eu stars are considered by [22], who argue that they have pure $r$-process origins. Are these stars enriched by both previous $s$-process and $r$-process events? At this point we simply cannot be sure. Further attempts to derive reliable $\mathrm{Ba}$ isotopes in other very metal-poor stars will be welcome, as will renewed efforts to increase the 


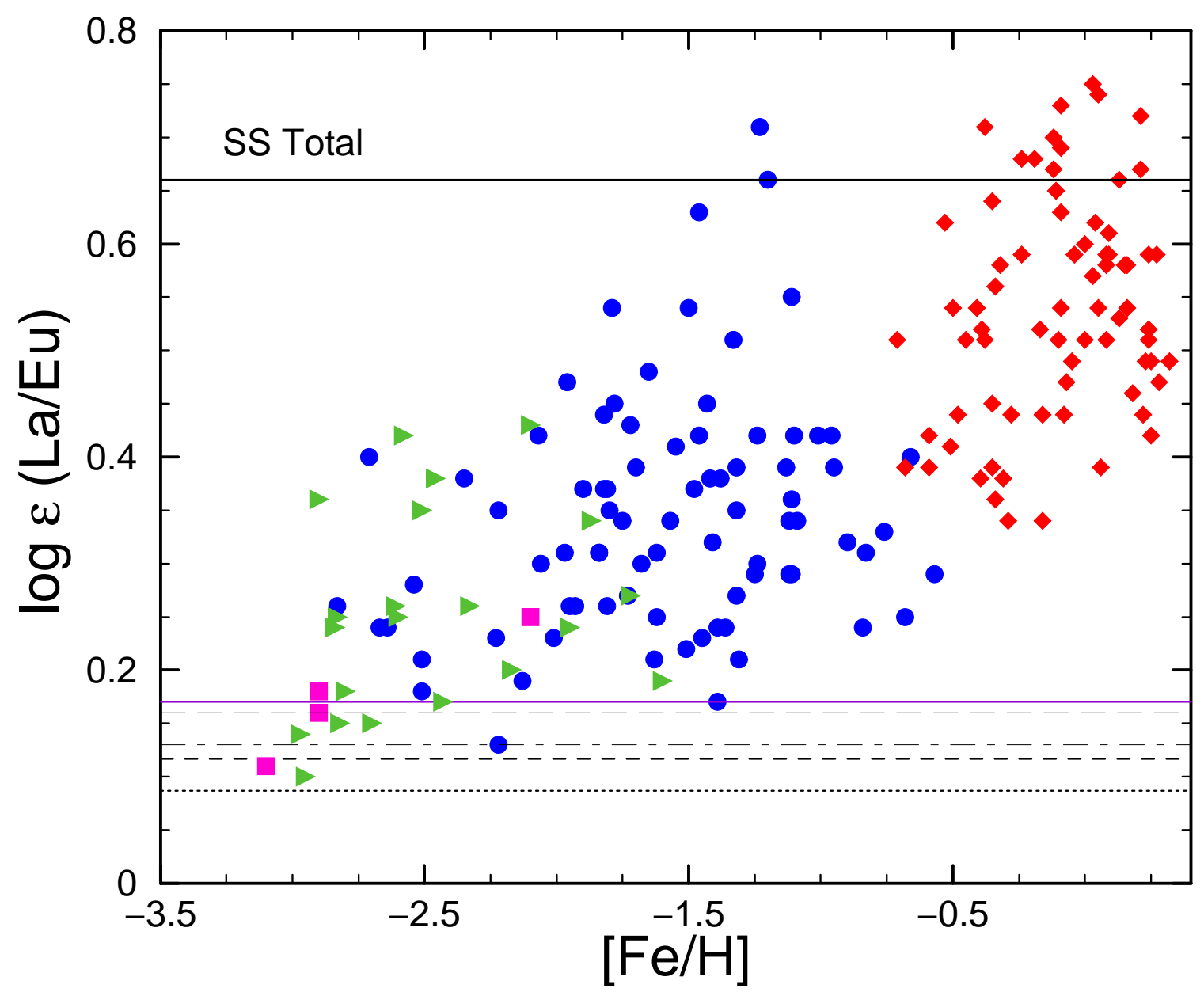

Figure 4: (Figure 16 of [26]) Abundance trends with respect to metallicity for the elemental ratio La/Eu in a large number of stars in our Galaxy. The filled blue circles are halo stars and the filled red diamonds are disk stars. The (green) right facing triangles are from [1]. The $r$-process-only ratios predicted for La/Eu in various literature sources are shown with black lines; see [26] for details of these predictions.

quality and quantity of Sm isotopic fractions first investigated by [17, 23].

The substantial growth of $n$-capture abundances in very metal-poor stars over the past couple of decades has illuminated the role of the $r$-process in early Galactic nucleosynthesis. But, happily, as old questions have been cleared up we have been presented with new puzzles that will need the future combined efforts of observations and theoreticians to answer well.

\section{References}

[1] Barklem, P., et al., The Hamburg/ESO R-process enhanced star survey (HERES). II. Spectroscopic analysis of the survey sample, Ast. \& Ap. 439 (2005) 129.

[2] Beers, T. C., Preston, G. W., \& Shectman, S. A., A search for stars of very low metal abundance. II Ast. J., 103 (1992) 1987. 
[3] Cowan, J. J., et al., The Chemical Composition and Age of the Metal-poor Halo Star BD $+17^{\circ} \hat{A} \check{r} 3248$, Ap. J. 572 (2002) 861.

[4] Cowan, J. J., et al., Hubble Space Telescope Observations of Heavy Elements in Metal-Poor Galactic Halo Stars, Ap. J. 627 (2005) 238.

[5] François, P., et al., First stars. VIII. Enrichment of the neutron-capture elements in the early Galaxy, Ast. \& Ap. 476 (2007) 935.

[6] Frebel, A., Christlieb, N., Norris, J. E., Thom, C., Beers, T. C., \& Rhee, J., Discovery of HE 1523-0901, a Strongly r-Process-enhanced Metal-poor Star with Detected Uranium, Ap. J. 660 (2007) L1117.

[7] Gallagher, A. J., Ryan, S. G., García Pérez, A. E., \& Aoki, W., The barium isotopic mixture for the metal-poor subgiant star HD140283, Ast. \& Ap. in press, astro-ph/10083541.

[8] Griffin, R., Gustafsson, B., Vieira, T., \& Griffin, R., HD 115444 - A barium star of extreme Population II, Mon. Not. Roy. Ast. Soc. 198 (1982) 637.

[9] Hayek, W., et al., The Hamburg/ESO R-process enhanced star survey (HERES). IV. Detailed abundance analysis and age dating of the strongly r-process enhanced stars CS 29491-069 and HE 1219-0312, Ast. \& Ap. 504 (2009) 511.

[10] Hill, V., et al., First stars. I. The extreme r-element rich, iron-poor halo giant CS 31082-001. Implications for the r-process site(s) and radioactive cosmochronology, Ast. \& Ap. 387 (2002) 560.

[11] Honda, S., Aoki, W., Ishimaru, Y., Wanajo, S., \& Ryan, S. G. Neutron-Capture Elements in the Very Metal Poor Star HD 122563, Ap. J. 643 (2006) 1087.

[12] Honda, S., Aoki, W., Ishimaru, Y., \& Wanajo, S,, Neutron-Capture Elements in the Very Metal-poor Star HD 88609: Another Star with Excesses of Light Neutron-Capture Elements, Ap. J. 666 (2007) 1189.

[13] Ivans, I. I., Simmerer, J., Sneden, C., Lawler, J. E., Cowan, J. J., Gallino, RR., \& Bisterzo, S., Near-Ultraviolet Observations of HD 221170: New Insights into the Nature of r-Process-rich Stars, Ap. J. 645 (2006) 613.

[14] Kratz, K.-L., Farouqi, K., Pfeiffer, B., Truran, J. W., Sneden, C., \& Cowan, John J., Explorations of the r-Processes: Comparisons between Calculations and Observations of Low-Metallicity Stars, Ap. J. 662 (2007) 39.

[15] Lambert, D. L., \& Allende Prieto, C., The isotopic mixture of barium in the metal-poor subgiant HD 140283, Mon. Not. Roy. Ast. Soc 335 (2002) 325.

[16] Lawler, J. E., Sneden, C., Cowan, J. J., Ivans, I. I., \& Den Hartog, E. A., Improved Laboratory Transition Probabilities for Ce II, Application to the Cerium Abundances of the Sun and Five r-Process-Rich, Metal-Poor Stars, and Rare Earth Lab Data Summary, Ap. J. Supp. 182 (2009) 51.

[17] Lundqvist, M., Wahlgren, G. M., \& Hill, V., Isotope structure of Sm II as an indicator of $r$ - vs. s-process nucleosynthesis, Ast. \& Ap. 463 (2007) 693.

[18] Malcheva, G., Blagoev, K., Mayo, R., Ortiz, M., Xu, H. L., Svanberg, S., Quinet, P., \& Biémont, E., Mon. Not. Roy. Ast. Soc. 367 (2006) 754.

[19] Magain, P., \& Zhao, G., Barium isotopes in the very metal-poor star HD 140283, Ast. \& Ap. 268 (1993) 27. 
[20] Mashonkina, L., Christlieb, N., Barklem, P. S., Hill, V., Beers, T. C., \& Velichko, A., The Hamburg/ESO R-process enhanced star survey (HERES). V. Detailed abundance analysis of the r-process enhanced star HE 2327-5642, Ast. \& Ap. 516 (2010) 46.

[21] Nilsson, H., Hartman, H., Engström, L., Lundberg, H., Sneden, C., Fivet, V., Palmeri, P., Quinet, P., \& Biémont, ÃL', Transition probabilities of astrophysical interest in the niobium ions $\mathrm{Nb}^{+}$and $\mathrm{Nb}^{2+}$, Ast. \& Ap. 511 (2010) 16.

[22] Roederer, I. U., Cowan, J. J., Karakas, A. I., Kratz, K.-L., Lugaro, M., Simmerer, J., Rarouqi, K., \& Sneden, C., The Ubiquity of the Rapid Neutron-Capture Process, Ap. J. in press, astro-ph/10094496.

[23] Roederer, I. U., Lawler, J. E., Sneden, C., Cowan, J. J., Sobeck, J. S., \& Pilachowski, C. A., Europium, Samarium, and Neodymium Isotopic Fractions in Metal-Poor Stars, Ap. J. 675 (2008) 723.

[24] Roederer, I. U., Sneden, C., Lawler, J. E., \& Cowan, J. J. New abundance determinations of Cadmium, Lutetium and Osmium in the r-process enriched star BD+17 3248., Ap. J. 714 (2010) L123.

[25] Simmerer, J., Sneden, C., Cowan, J. J., Collier, J., Woolf, V. M., \& Lawler, J. E., The Rise of the s-Process in the Galaxy, Ap. J. 617 (2004) 1091.

[26] Sneden, C., Cowan, J. J., \& Gallino, R., Neutron-capture elements in the early galaxy, Ann. Rev. Ast. Ap. 46 (2008) 241.

[27] Sneden, C., et al., The Extremely Metal-poor, Neutron Capture-rich Star CS 22892-052: A Comprehensive Abundance Analysis, Ap. J. 591 (2003) 936.

[28] Sneden, C., Lawler, J. E., Cowan, J. J., Ivans, I. I., \& Den Hartog, E. A. New rare earth element abundance distributions for the Sun and five r-process-rich very metal-poor stars, Ap. J Supp. 182 (2009) 182.

[29] Wanajo, S., Nomoto, K., Janka, H.-T., Kitaura, F. S., \& MÃijller, B., Nucleosynthesis in Electron Capture Supernovae of Asymptotic Giant Branch Stars, Ap. J. 695 (2009) 208.

[30] Westin, J, Sneden, C., Gustafsson, B., \& Cowan, J. J., The r-Process-enriched Low-Metallicity Giant HD 115444, Ap. J. $\mathbf{5 3 0}$ (2003) 783. 